\title{
Tongue Tie (Ankyloglossia)
}

\author{
Osama Mohamed Aldebei* \\ King Hussein Medical Center, Otorhinolaryngology Surgery, Jordan
}

Submission: June 24, 2016; Published: July 25, 2016

*Corresponding author: Osama Mohamed Aldebei, King Hussein Medical Center, Otorhinolaryngology Surgery, Royal Medical Services, As salt P.O BOX (716), Amman, Jordan, Tel: 00962772463300; Email: aldebeiosama@yahoo.com

\begin{abstract}
Objectives: To explore the beliefs and practices of surgeons regarding surgical intervention to release tongue tie, incidence, and clinical significance and to determine the main referral sources.

Methods: 1618 patients were received as they had been referred to oral and maxillofacial surgery clinic to have ankyloglossia. They were examined using the guidelines described by Fletcher and Daly15. The age, sex, referring source and cause were recorded. Results: 307 (19\%) were judged not to have ankyloglossia. The others 1311 (81\%) were examined for any speech problem, feeding difficulty or mechanical-social issue. then surgical treatment offered. Only few patients had experienced a problem regarding speech 26 (2\%), feeding 43 (3.3\%) or mechanical issues $13(1 \%)$. And only in very few situations an improvement was reported regarding speech 19 (1.4\%).
\end{abstract}

Conclusion: Ankyloglossia is not an uncommon problem with a very low clinical significance.

Keywords: Ankyloglossia; Tongue tie

\section{Introduction}

Tongue-tie or ankyloglossia is a congenital oral anomaly defined as 'a condition in which the tip of the tongue cannot be protruded beyond the lower incisor teeth because of a short frenulum'. Tongue-tie was reported to be more prevalent in males than females with a ratio of 2.6:1.0. Although its existence has been recognized for centuries, the clinical significance (from diagnosis to management) remains controversial [1]. Opinions range widely regarding the significance and treatment of this anomaly; some feel that ankyloglossia is only rarely symptomatic, while others feel not [2-4]. Possible consequences of ankyloglossia include infant breast-feeding difficulties, speech disorders and various mechanical and social issues such as an inability to lick the lips, play a wind instrument and 'French kiss' [1,5-10]. Historically, tongue ties were thought to affect breastfeeding and were thus divided [11-14]. Today, many current textbooks and articles state that tongue ties rarely, if ever, impede feeding or speech, but this assertion is not evidence based [6]. The current study was conducted to study the incidence, current beliefs, clinical significance and treatment of ankyloglossia.

\section{Patient's and Methods}

1618 patients were referred to oral and maxillofacial surgery clinic over seven years practice at Jordanian military hospitals with an age range from (<one day-sixteen years) from different specialties including pediatric clinic, ENT clinic and general dental practitioner clinic or self referral. 1152 were males and 466 females with a male to female ratio 2.5:1. All patients underwent an oral examination, with qualitative grading of the degree of ankyloglossia as mild, moderate or severe. After examination 1311patients were judged to have ankyloglossia. 307 patients were not found to have any type of ankyloglossia. In our study we used the guidelines described by Fletcher and Daly15 for qualitative grading of the degree of ankyloglossia; their guidelines included inability of the tongue tip to elevate to contact the upper alveolar ridge or to protrude beyond the lower gum, restriction of side-to-side motion, and the presence of a notched tongue.

Criteria for establishing the diagnosis of ankyloglossia range from simple (e.g., the tip of the tongue cannot be protruded beyond the lower incisor teeth) [1] to more complex and cumbersome ratios of various lingual dimensions [15]. The use of tongue protrusion and elevation measurements assists in the clinical assessment of ankyloglossia and provides an effective means for documenting preoperative and postoperative lingual mobility. All patients were screened for any problem regarding speech, feeding or mechanical-social issues.

All the patients had surgical treatment either Frenotomy or Frenuloplasty.

\section{Results}

\section{Incidence of ankyloglossia}

1311 patients were identified as having tongue tie, 952 males and 359 females. Ankyloglossia is not uncommon, seeing almost 
190 cases annually in our practice. With a male to female ratio of 2.7:1. In other studies ankyloglossia occurs in approximately $5 \%$ of newborn infants, at a male to female ratio of 2.6:1. On oral examination (n_ 1311), 353 (27\%) patients were judged to have mild, 864 (66\%) moderate, and 94 (7\%) severe ankyloglossia.

\section{Reasons for referral}

Physicians recommendations specially the pediatricians (52\%).

\section{Parents fears (38\%).}

Speech disorders without referral (2\%).

Relative's advice (8\%).

In general most of the patients were seeking treatment cause of speech problems or in fact a fear of speech disorder even in the future. None of the patients were referred cause of feeding or mechanical and social issues problems.

\section{Consequences of ankyloglossia}

In our experience ankyloglossia rarely causes feeding difficulties, but tongue-tie is at least sometimes associated with a speech disorder, in our study 26 (2\%) patients reported a speech problem and in 19 patients, the parents reported improvement of phonetic problems after surgery although we couldn't notice that. Feeding problems was reported by $43(3.3 \%)$ mothers, namely painful sore nipple and ineffective sucking. Social: mechanical issues such as inability to lick the lips or play a wind instrument, is rarely encountered $13(1 \%)$.

\section{Surgical treatment}

The criteria used to decide surgical treatment was:

- Patient's inability to lick his/her lowers lips.

- $\quad$ Notched tongue.

- $\quad$ Speech disorders.

- $\quad$ Mild degree of tongue tie with parent's request or if associated with feeding difficulty.

The timing of surgery was dependent on the time of presentation to the clinic although we believe that early intervention when it is indicated is better. Cause in some cases surgery is less effective when undertaken in older children or adults that are after speech problems appear. And in this situation you might be in need for an adjunct speech therapist. Frenuloplasty and frenotomy were the used techniques either at the operation room or at the outpatient clinic depending on the age and general medical condition of the patients.

\section{Outcome}

A high rate of success (73\%) was encountered with the indication of speech disorder (as parents reported), mechanical: social issues (92\%) and sucking: feeding dysfunction (70\%) (After one month postoperatively). Two cases of the older age group were in need for speech therapy after surgery cause the improvement was not complete within three months after surgery.

\section{Complications}

The complications following surgery either frenotomy or frenuloplasty was extremely rare. The most common complication is residual tongue-tie or recurrent ankyloglossia due to scarring which was encountered in $5(0.4 \%)$ patients; however this tonguetie is usually less severe than the original one. Prolonged bleeding was encountered in $2(0.2 \%)$ cases. Presence of new speech disorder or worsening was encountered in one patient (age: 15 year old). Infection or increased feeding difficulty as a result of Frenotomy: Frenuloplasty were never encountered.

\section{Discussion}

Ankyloglossia is relatively uncommon. The incidence of ankyloglossia reported in the literature ranges from $0.02-4.4 \%$ [16]. This variation in reported incidence may be due to lack of a uniform definition and objective grading system for tonguetie, as well as the possibility of spontaneous resolution with age. Tongue-tie is thought to be less common in adults as compared to infants [10]. There have been numerous attempts to define tongue-tie. These include grading the shortness of the frenulum (mild, moderate and severe) as well as noting thickness (thin or thick frenulum) and notching of the tongue [17]. However, no one approach has been universally adopted and undergone validation, nor to our knowledge has any classification system been the subject of any inters or intra judge reliability. The identification of tongue-tie therefore remains a subjective determination made by a variety of clinicians including surgeons, speech pathologists, midwives, lactation consultants and pediatricians.

The lack of a uniform grading system has complicated the study of ankyloglossia, making it difficult to compare reports in the literature and difficult to assess the need for and results of treatment. Until the present, perhaps the most clinically useful guide for establishing the presence of ankyloglossia was a qualitative one, as described by Fletcher and Daly [11]; their guidelines included inability of the tongue tip to elevate to contact the upper alveolar ridge or to protrude beyond the lower gum, restriction of side-to-side motion, and the presence of a notched tongue.

Most of the medical and nursing literature stated that tongue ties did not impede breastfeeding $[1,18,19]$. However, ankyloglossia has been reported to make breastfeeding difficult, causing sore nipples, poor infant weight gain and early weaning $[6,9,16,20-22]$. Three prospective studies have assessed the indications and outcome of tongue tie division in infants with feeding difficulties, all advocating division in symptomatic infants. With the realization that breastfeeding confers many advantages there is an increased expectation that mothers will breastfeed.

At presentation, three common symptoms were associated with breastfeeding problems caused by a tongue tie: latching on, 
nipple trauma and continuous feeding. During breastfeeding, the nipple becomes elongated, up to three times its normal size, and stretches back to the junction of the hard and soft palate. The lactiferous sinuses are massaged by the tongue and lower alveolar ridge. If the tongue is tethered, the nipple is squeezed by the upper and lower gums (chomp). This is inefficient, is traumatic, can be very painful, and does not allow normal breastfeeding to occur. Both mother and baby become increasingly frustrated and formula feeding often results. Some mothers try expressing and cup- and bottle-feeding or use a nipple shield. Careful repositioning with lactation consultant input allows some mothers to breastfeed adequately. Sometimes, the new position is very unusual (e.g., holding the infant vertically upright). In our study we never encounter an objective evidence to support this idea although $43(3.3 \%)$ of the mothers reported painful sore nipple and ineffective sucking.

The concept of tongue-tie affecting speech is also not new. Historical references to tongue-tie may be found beginning in biblical times. In the older infant, toddler and young child, poor speech has been frequently listed as a consequence of tonguetie, suggesting that, if identified, a tongue-tie should be released surgically to prevent the development of later speech difficulties. In older children and adolescents, speech problems, the inability to protrude the tongue, lick the lips or an ice-cream as well as indulge in tongue kissing were cited as reasons for surgical intervention.

However, the effect of ankyloglossia on speech remains controversial the majority believe that surgical therapy is frequently or always helpful for this condition. There is a huge controversy regarding the timing of surgical repair for ankyloglossia. Some believe that it is appropriate at any age while others believe that it is not appropriate for young infants or children older than twelve years. Similarly, while some authors in the literature have advocated repair in infancy and prior to the development of speech [20-22] others advocate waiting until a speech problem becomes manifest, usually after the age of four years $[1,5,8]$.

Unfortunately, there is no method for predicting which patients with tongue-tie are likely to become symptomatic that complicates decision-making regarding surgical timing. Complications reported historically for Frenotomy and Frenuloplasty are few, and include infection, excessive bleeding, recurrent ankyloglossia due to scarring, one case of a new speech disorder developing postoperatively, and 'tongue swallowing' due to excessive tongue mobility $[1,2,5,9]$.

The most common complication reported in this study was recurrent ankyloglossia due to scarring or inadequate primary surgery in which we noticed that frenotomy was the used surgical technique. The literature suggests that Frenuloplasty, as opposed to frenotomy, may decrease the risk of recurrence.

\section{Conclusion}

Ankyloglossia is not an uncommon problem and although surgical release of ankyloglossia is routinely performed at our department it has a very low clinical significance, we highlighted the controversial nature of ankyloglossia. There is currently no consensus among surgeons, pediatricians, lactation consultants and speech pathologists regarding the significance and management of ankyloglossia.

\section{References}

1. JL Paradise (1990) Evaluation and treatment for ankyloglossia, JAMA 262: 2371.

2. M. Ulshen (1996) Clinical manifestations of gastrointestinal disease. In: Behrman RE, et al. (Eds.), Nelson Textbook of Pediatrics, $\left(15^{\text {th }}\right.$ edn), W.B. Saunders Co, Philadelphia, USA, pp. 1031.

3. Horton CE, Crawford HH, Adamson JE, Ashbell TS (1969) Tongue-ti. Cleft Palate J $6: 8-23$.

4. Marmet C, Shell E, Marmet R (1990) Neonatal frenotomy may be necessary to correct breastfeeding problems. J Hum Lact 6(3): 117121.

5. Fletcher SG, Meldrum JR (1968) Lingual function and relative length of the lingual frenulum. J Speech Hearing Res 11(2): 382-390.

6. Wright JE (1995) Tongue-tie. J Paediatr Child Health 31(4): 276-278.

7. Fleiss PM, Burger M, Ramkumar H, Carrington P (1990) Ankyloglossia: a cause of breastfeeding problems? J Hum Lact 6(3): 128-129.

8. Eminent Physician (1959) The Nurses Guide or the Right Method of Bringing Up Young Children. 1729. Cited by: Cullum IM. An old wives' tale. Br Med J 2: 497-498.

9. Pechey JA (1959) General Treatise of the Diseases of Infants and Children. London; 1697:91-93. Cited by: Cullum IM. An old wives' tale. Br Med J 2:497-498.

10. Moss W (1959) An Essay on the Management, Nursing and Diseases of Children. ( $2^{\text {nd }}$ edn). London; 1794. Cited by: Cullum IM. An old wives' tale. Br Med J 2:497-498.

11. Fletcher SG, Daly DA (1974) Sublingual dimensions in infants and young children. Arch Otolaryngol 99(4): 292-296.

12. Messner AH, Lalakea ML, Aby J, Macmahon J, Bair E (2000) Ankyloglossia: incidence and associated feeding difficulties. Arch Otolaryngol Head Neck Surg 126(1): 36-39.

13. Catlin FI (1971) Tongue-tie. Arch Otolaryngol 94(6): 548-557.

14. Notestine GE (1990) The importance of the identification of ankyloglossia (short lingual frenulum) as a cause of breastfeeding problems. J Hum Lact 6(3): 113-115.

15. Berg KL (1990) Tongue-tie (ankyloglossia) and breastfeeding: a review. J Hum Lact 6(3): 109-112.

16. G Wall. Consultants' Corner. J Hum Lact 6: 122-127.

17. Masaitis NS, Kaempf JW (1996) Developing a frenulotomy policy at one medical centre: a case study approach. J Hum Lact 12(3): 229-232.

18. American Academy of Pediatrics Working Group on Breast Feeding (1997) Breastfeeding and the role of human milk. Pediatrics 6: 10351039.

19. Singhal A, Cole TJ, Lucas A (2001) Early nutrition in pre-term infants and later blood pressure: 2 cohorts after randomized trials. Lancet 357(9254): 413-419. 
20. Messner AH, Lalakea ML (2002) The effect of ankyloglossia on speech in children. Otolaryngol Head Neck Surg 127(6): 539-545.

21. Horton CE, Crawford HH, Adamson JE, Ashbell TS (1971) Tongue tie. Arch Otolaryngol 94: 548-557.
22. Warden PJ (1991) Ankyloglossia: A review of the literature. Gen Dent 39(4): 252-253. 